INPLASY

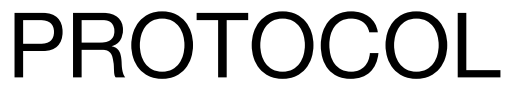

To cite: Zeng et al. Diagnostic ability of DCE-MRI for prostate cancer and clinically significant prostate cancer in equivocal lesions: A Systematic Review and Meta-analysis. Inplasy protocol 202090002. doi: 10.37766/inplasy2020.9.0002

Received: 01 September 2020

Published: 01 September 2020

Corresponding author: Liangping Luo

tluolp@jnu.edu.cn

Author Affiliation:

The First Affiliated Hospital of Jinan University

Support: None.

Review Stage at time of this submission: Formal screening of search results against eligibility criteria.

Conflicts of interest: None.

\section{Diagnostic ability of DCE-MRI for prostate cancer and clinically significant prostate cancer in equivocal lesions: A Systematic Review and Meta-analysis}

Zeng, J1; Fan, M2; Xiao, Z3; Cheng, Q4; Shi, C5; Luo, L6.

Review question / Objective: The aim of this meta-analysis is to explore the diagnostic ability of DCE-MRI for prostate cancer and clinically significant prostate cancer in equivocal lesions (PIRADS 3 or likert scale of 1-5 category 3 lesions). Condition being studied: Prostate cancer ranks as the second most frequent cancer and the fifth leading cause of cancer death in men. It is the most commonly diagnosed cancer among men in more than half of the countries in the world MRI is now an established tool for the diagnosis of prostate cancer, with promising future, and pre-biopsy MRI is increasingly being performed in patients with elevated PSA. Currently, whether to include DCE-MRI in prostate MRI or not is a controversial subject and many original articles about the comparison between bp-MRI and mp-MRI are being published.

INPLASY registration number: This protocol was registered with the International Platform of Registered Systematic Review and Meta-Analysis Protocols (INPLASY) on 01 September 2020 and was last updated on 01 September 2020 (registration number INPLASY202090002).

\section{INTRODUCTION}

Review question / Objective: The aim of this meta-analysis is to explore the diagnostic ability of DCE-MRI for prostate cancer and clinically significant prostate cancer in equivocal lesions (PIRADS 3 or likert scale of 1-5 category 3 lesions).
Condition being studied: Prostate cancer ranks as the second most frequent cancer and the fifth leading cause of cancer death in men. It is the most commonly diagnosed cancer among men in more than half of the countries in the world MRI is now an established tool for the diagnosis of prostate cancer, with promising future, and pre-biopsy MRI is increasingly being 
performed in patients with elevated PSA. Currently, whether to include DCE-MRI in prostate MRI or not is a controversial subject and many original articles about the comparison between bp-MRI and $\mathrm{mp}$ MRI are being published.

\section{METHODS}

Participant or population: The following patients were considered as participants: (1) patients with clinical suspicion of $\mathrm{PCa}$ based on elevated prostate-specific antigen (PSA) or an abnormal digital rectal examination (DRE); (2) patients neither surgery nor chemotherapy was conducted before magnetic resonance imaging (MRI).

Intervention: Dynamic contrast-enhanced magnetic resonance imaging is the mian intervention.

Comparator: Pathological results are the main comparator.

Study designs to be included: Randomized controlled trials will be included.

Eligibility criteria: Retrospective and prospective studies undergoing prostate MR imaging in whom there were reported equivocal lesions (PIRADS 3 OR 5-likert 3) identified by prostate MR imaging. Studies in which only compared DWI with DWI+DCE-MRI outcomes were excluded.

Information sources: Electronic databases (pubmed and embase), contact with authors.

Main outcome(s): PCa and csPCa detection in patients with category 4 lesions upgraded from category 3 lesions in PIRADS or a likert scale of 1-5 due to positive DCE findings.

Quality assessment / Risk of bias analysis: We assessed the methodological quality of the included studies by using the revised instrument for the Quality Assessment of Diagnostic Accuracy Studies-2 tool(QUADAS-2). RevMan software (version 5.3) was used to assess the quality of studies.
Strategy of data synthesis: The coupled forest plots for the sensitivity and specificity were drew. Summary receiver operating characteristic curve (SROC) and area under the SROC curve (AUC) were constructed to summarize the diagnostic accuracy. Cochran's $Q$ test and the inconsistency index (I2value) were used to assess the heterogeneity of the included studies, with $\mathbf{P}<0.05$ or $12>50 \%$ indicating the presence of substantial heterogeneity. When $12>50 \%$, the random-effects model of DerSimonian and Laird was used for meta-analysis and a fixed-effect model was applied for $12<50 \%$. Meta-regression analyses were also performed to study the causes of heterogeneity. Egger's Funnel plot analysis for determining the publication bias was conducted. The statistical analyses were performed using Stata software (version 12.0, StataCorp).

Subgroup analysis: We will consider subgroups such as study design and magnetic field strength.

Sensibility analysis: If obvious heterogeneity was noted, sensitivity analysis will be performed.

Country(ies) involved: China.

Keywords: Clinically significant Prostate cancer; DCE-MRI; PI-RADS; a likert scale of 1-5.

Contributions of each author:

Author 1 - Jing Zeng.

Author 2 - Meng Fan.

Author 3 - Zeyu Xiao.

Author 4 - Qingqing Cheng.

Author 5 - Changzheng Shi.

Author 6 - Liangping Luo. 